\title{
Simultaneous translation on two rods improves the correction and apex translocation in adolescent patients with hypokyphotic scoliosis
}

\author{
Daisuke Sakai, MD, PhD, Jordy Schol, MSc, Akihiko Hiyama, MD, PhD, Hiroyuki Katoh, MD, PhD, \\ Masahiro Tanaka, MD, PhD, Masato Sato, MD, PhD, and Masahiko Watanabe, MD, PhD \\ Department of Orthopaedic Surgery, Tokai University School of Medicine, Kanagawa, Japan
}

\begin{abstract}
OBJECTIVE The objectives of this study were to apply the simultaneous translation on two rods (ST2R) maneuver involving rods contoured with a convexity at the desired thoracic kyphosis (TK) apex level and to assess the effects on the ability to support triplanar deformity corrections, including TK apex improvement, in patients with hypokyphotic adolescent idiopathic scoliosis (AIS).
\end{abstract}

METHODS Using retrospective analysis, the authors examined the digital records that included 2- to 4-week, 1-year, and 2-year postoperative radiographic follow-up data of female hypokyphotic (TK < $20^{\circ}$ ) AIS patients (Lenke type 1-3) treated with ST2R. The authors assessed the corrections of triplanar deformities by examining the main Cobb angle, TK, rib hump, apical vertebral rotation, Scoliosis Research Society 22-item questionnaire scores, and TK apex translocation. In order to better grasp the potential of ST2R, the outcomes were compared with those of a historical matched casecontrol cohort treated with a standard rod rotation (RR) maneuver.

RESULTS Data were analyzed for 25 AIS patients treated with ST2R and 27 patients treated with RR. The ST2R group had significant improvements in the main Cobb angle and TK, reduction in the rib hump size at each time point, and a final correction rate of $72 \%$. ST2R treatment significantly increased the kyphosis apex by an average of 2.2 levels. The correction rate was higher at each time point in the ST2R group than in the RR group. ST2R engendered favorable TK corrections, although the differences were nonsignificant, at 2 years compared with the RR group $(p=0.056)$. The TK apex location was significantly improved in the ST2R cohort $(p<0.001)$. At the 1-month follow-up, hypokyphosis was resolved in $92 \%$ of the ST2R cohort compared with $30 \%$ of the RR cohort.

CONCLUSIONS Resolving hypokyphotic AIS remains challenging. The ST2R technique supported significant triplanar corrections, including TK apex translocation and restoration of hypokyphosis in most patients. Comparisons with the RR cohort require caution because of differences in the implant profile. However, ST2R significantly improved the coronal and sagittal corrections. It also allowed for distribution of correctional forces over two rod implants instead of one, which should decrease the risk of screw pullout and rod flattening. It is hoped that the description here of commercially available reducers used with the authors' surgical technique will encourage other clinicians to consider using the ST2R technique. https://thejns.org/doi/abs/10.3171/2020.8.SPINE201232

KEYWORDS hypokyphosis; spinal deformity; surgical technique; adolescent idiopathic scoliosis; surgery

A DOLESCENT idiopathic scoliosis (AIS) is a triplanar spine deformity that involves abnormal curvatures and vertebral rotation and is estimated to affect $0.5 \%-5.2 \%$ of adolescents. ${ }^{1}$ Contemporary treatments include surgical intervention to restore the spine by reducing the magnitude of the abnormal alignment. These interventions aim to improve spine stability with minimal impairment of mobile segments, reduce neurological risk, prevent deformity progression, and improve patient selfimage and physical function while reducing pain.

The outcomes of surgical correction techniques have improved considerably in recent decades. The single rod rotation (RR) technique ${ }^{2}$ is a commonly used intervention and is hypothesized to work by inducing vertebral column derotation along the transverse axis. ${ }^{3}$ Using anchor points in the vertebrae, a curved rod is placed onto the concav-

ABBREVIATIONS AIS = adolescent idiopathic scoliosis; $\mathrm{AVR}=$ apical vertebral rotation; $\mathrm{CCI}=$ Cincinnati correction index; $\mathrm{LL}=$ lumbar lordosis; $\mathrm{RH}=$ rib hump; $\mathrm{RR}=$ rod rotation; SRS-22r = Scoliosis Research Society 22-item questionnaire; ST2R = simultaneous translation on two rods; TK = thoracic kyphosis.

SUBMITTED July 8, 2020. ACCEPTED August 10, 2020.

INCLUDE WHEN CITING Published online February 5, 2021; DOI: 10.3171/2020.8.SPINE201232. 
ity and is forcefully rotated toward the convex aspect to provoke a rotational correction. Contemporary RR adaptations include the use of pedicle screws and have enabled impressive coronal and axial plane corrections. However, these adaptations remain unable to engender appropriate corrections in the sagittal plane in some cases, including correction of thoracic kyphosis (TK) and of the TK apex, a particular shortcoming for hypokyphotic AIS patients. In general, vertebral rotation techniques lower the TK apex, which relocates the apex to the most derotated vertebral level. ${ }^{4}$

Considering that the thoracic coronal curve apex is located lower than the sagittal thoracic apex, reduction of TK is associated with spinal imbalance and functional deterioration in adults with spinal deformity, ${ }^{5}$ and TK maintenance is required for the preservation of lumbar lordosis (LL) in AIS patients. ${ }^{6}$ Moreover, a retrospective review of 352 adult spinal deformity patients concluded that a positive sagittal balance is associated with symptom severity and that kyphosis is better tolerated in the upper thoracic regions. ${ }^{7,8}$ Similarly, in AIS patients, TK has been shown to strongly affect cervical and sagittal alignment. ${ }^{9}$ The RR technique is associated with an increase in rib hump (RH) size and may not be efficient for patients presenting with a larger deformity or a relatively rigid spine. ${ }^{3}$

Clement et al. were the first to demonstrate the ability to restore thoracic hypokyphosis $\left(<20^{\circ}\right)$ by applying the simultaneous translation on two rods (ST2R) maneuver. ${ }^{10}$ In their first retrospective study, these authors showed the ability of ST2R to improve TK, with improvements remaining through the final follow-up..$^{10}$ Notably, the ST2R technique was able to restore TK to $>20^{\circ}$ for all 14 hypokyphotic AIS patients compared with $70 \%$ of patients treated with a cantilever reduction technique. ${ }^{10} \mathrm{~A}$ later study of exclusively thoracic hypokyphotic AIS patients reported an average final $\mathrm{TK}$ of $32^{\circ}$ and a $\mathrm{TK}>20^{\circ}$ in all 24 participants. ${ }^{11}$ Although the development of the ST2R technique allowed for successful TK corrections, including correction of hypokyphosis, it appears unable to control the TK apex level translocation. The role of the TK apex in maintaining the sagittal balance and affecting the quality of life is not well understood. It is known that the apex has a major influence on the shape of the back, which determines the posture and is thus likely to affect important factors such as self-image and disability, ${ }^{12}$ which is complemented by non-AIS-specific deformity correction studies suggesting that apex improvements are associated with enhanced clinical outcomes. ${ }^{12,13}$

A recent retrospective study of 36 AIS patients by Faldini et al. ${ }^{4}$ described an ST2R adaptation involving asymmetrically contoured rods fitted to the desired sagittal shape, including a convexity at the desired apex level. The authors reported significant improvements in the TK angle and overall quality of life and noted that their technique allows for the manipulation of the TK apex level to a more desired height. Although the work of Faldini et al. ${ }^{4}$ is promising, they did not quantify the TK apex alterations or include a control group, which makes interpretation of their effectuated apex corrections difficult. Additionally, their study did not focus specifically on hypokyphotic AIS. As such, there is a need to compare the results of the
ST2R technique to those obtained with other commonly used correction maneuvers and to assess the ability to correct the TK apex position.

Here, we present the results of our retrospective study, in which we assessed the potential of a modified ST2R technique enhanced by rods specifically contoured to present a convexity at the desired TK apex level, to produce triplanar curvature corrections. We focused on the ability of this technique to resolve hypokyphosis and to engender TK and TK apex corrections. To examine further the potential of the ST2R technique, we included a historical matched case-control cohort treated with a standard RR maneuver and compared the respective correctional outcomes.

\section{Methods}

\section{Patient Selection}

To assess the ability of the ST2R technique to improve the sagittal profile correction and TK apex translocation in hypokyphotic AIS patients, we compared our ST2R cohort to a historical RR-treated cohort. All procedures described in this study were performed in accordance with the ethical standards directed by and upon approval of the Committee on Ethics and the Institutional Review Board of Tokai University School of Medicine (12R-036, 16R-052) and in accordance with the 1964 Declaration of Helsinki and its later amendments. Informed consent was obtained from the patients included or was collected from a parent or legal guardian of participants younger than 18 years. In total, 52 female patients with AIS with a TK < $20^{\circ}$ were included. Specifically, this involved an ST2R cohort, which included 25 patients subjected to ST2R, treated from April 2014 to December 2017, and a historical control group with 27 subjects treated by RR from January 2011 until April 2014. Patients were case matched and selected according to the main Cobb angle in the thoracic region T2-12 and were mainly classified as Lenke type 1 (Table 1).

\section{Surgical Procedure}

All surgeries were performed by the same spine surgeon (D.S.). Under general anesthesia and continuous intraoperative monitoring, all lateral aspects of the vertebral processes and the spinous processes for vertebra levels scheduled to be manipulated were exposed via a standard posterior midline incision. Fusion levels were determined based on the work of Lenke et al. ${ }^{14}$ The surgical navigation system (e.g., StealthStation S7, Medtronic) and CT imaging were applied for careful visualization of the screw angles and placement. For the ST2R technique, with the use of live navigation tools, the angle, width, and length of the pedicle screws inserted via the process were determined for each vertebra. After confirmation, uniplanar pedicle screws were inserted into each vertebra scheduled for manipulation (Fig. 1A). Next, depending on the extent of regional spine stiffness and the intended correction, the inferior, superior, and spinous articular processes were excised (Fig. 1B). When desired, the excised bone specimens were preserved and later applied for autologous bone grafting. The screws were then tightly secured and tulip- 
TABLE 1. Baseline clinical and radiographic values

\begin{tabular}{|c|c|c|c|}
\hline & $\mathrm{RR}$ & ST2R & p Value \\
\hline Participants, no. & 27 & 25 & \\
\hline \multicolumn{4}{|l|}{ Lenke type } \\
\hline 1 & $24(89 \%)$ & $22(88 \%)$ & $0.94^{*}$ \\
\hline II & $2(7 \%)$ & $2(8 \%)$ & \\
\hline III & $1(4 \%)$ & $1(4 \%)$ & \\
\hline Age, yrs & $14.1 \pm 2.4(10-19)$ & $14.8 \pm 2.1(11-20)$ & $0.34 \dagger$ \\
\hline Height, cm & $153.7 \pm 6.0(136.5-164.8)$ & $156.2 \pm 7.3(141.0-169.0)$ & $0.17 \dagger$ \\
\hline Weight, kg & $46.4 \pm 4.6(33.0-54.7)$ & $45.3 \pm 7.5(29.0-65.0)$ & $0.53 \dagger$ \\
\hline Arm span, cm & $154.8 \pm 9.2(133-168)$ & $155.8 \pm 8.5(140-170)$ & $0.73 \dagger$ \\
\hline Risser grade & $3.7 \pm 1.2(0-5)$ & $3.9 \pm 0.9(2-5)$ & $0.78^{*}$ \\
\hline Main thoracic Cobb angle, ${ }^{\circ}$ & $55.3 \pm 8.7(45-74)$ & $56.8 \pm 9.8(43-75)$ & $0.57 \dagger$ \\
\hline Active bending, ${ }^{\circ}$ & $32.9 \pm 12.0(12.5-64.0)$ & $36.1 \pm 12.3(13.4-60.0)$ & $0.35 \dagger$ \\
\hline Flexibility, \% & $41.2 \pm 17.1(8.5-72.0)$ & $37.5 \pm 14.2(15-70.0)$ & $0.40 \dagger$ \\
\hline $\mathrm{TK}^{\circ}$ & $10.2 \pm 6.8(-8$ to 18$)$ & $9.4 \pm 7.1$ (-8 to 20$)$ & $0.67 \dagger$ \\
\hline $\mathrm{LL},{ }^{\circ}$ & $49.0 \pm 13.8(15.5-74)$ & $48.8 \pm 12.0(10-66)$ & $0.96 \dagger$ \\
\hline $\mathrm{AVR}^{\circ}{ }^{\circ}$ & $17.0 \pm 7.5(7.5-36)$ & $16.4 \pm 6.7(6.1-30)$ & $0.78 \dagger$ \\
\hline $\mathrm{RH}, \mathrm{cm}$ & $14.2 \pm 4.6(5-22)$ & $13.5 \pm 4.8(5-25)$ & $0.62 \dagger$ \\
\hline TK apex level & $7.9 \pm 1.0(10-7)$ & $8.2 \pm 0.9(9-7)$ & $0.15^{*}$ \\
\hline
\end{tabular}

Baseline demographic and radiographic data for the adolescent patients with hypokyphotic AIS treated with the standard RR or our adapted ST2R technique. Values are presented as number (\%) of patients or mean \pm SD (range) unless otherwise indicated.

* Mann-Whitney U-test.

† Student t-test.

screw heads were realigned to ensure proper orientation for rod insertion. Next, sagittal and coronal radiographic images were obtained to confirm appropriate screw insertion.

Upon confirmation, commercially available reducers (e.g., Rockets Threaded Reducers, Zimmer Biomet; Deformity Crickets, K2M, Inc.; or SMARTLINK, Medtronic) were attached onto the pedicle screwheads (Figs. 1C, 2A, and $3 \mathrm{~A})$. Each reducer type was applied and confirmed to be effective (data not shown). Either short or long re-
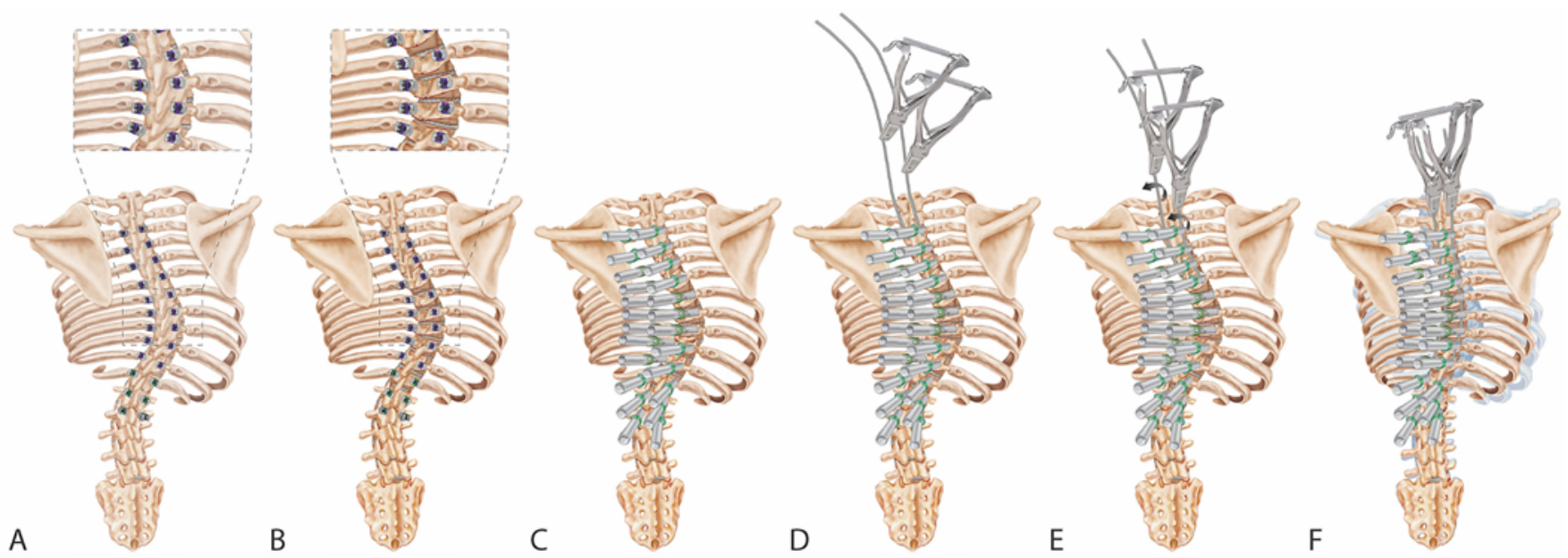

FIG. 1. Preparation and simultaneous prebent rod insertion. Graphic representation of simultaneous translation with the dual contoured rod (ST2R) technique presented from a posterior view. A: Pedicle screws are bilaterally inserted in the vertebra pedicles along the spine levels scheduled for corrections. B: Depending on spine rigidity and intensity of deformity, region-specific transverse, superior, and spinous articular processes are excised. C: Reducers are attached to each inserted pedicle screw. D: Two rods, shaped with the TK apex at T4-6 in order to translate the apex from the general area of T8-9 toward the upper region, are bilaterally and simultaneously inserted along the reducer canals. E: Rotating the rod will enable insertion of the rods through the reducer canals. F: The insertion of the rods will mildly induce axial and coronal corrections. Finally, in the next step (see Fig. 2), the rods are rerotated to match the sagittal profile, in order to perform the next final sagittal and coronal plane corrections. Copyright Daisuke Sakai. Published with permission. 
A

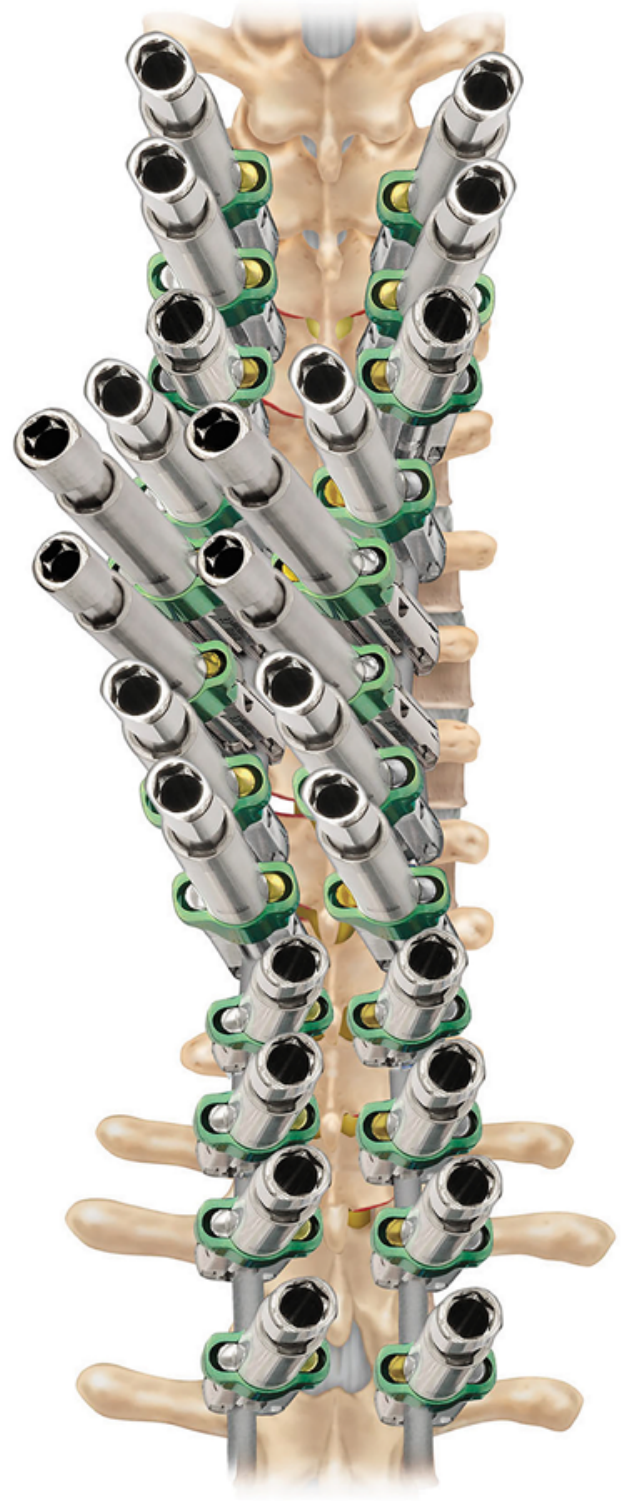

B
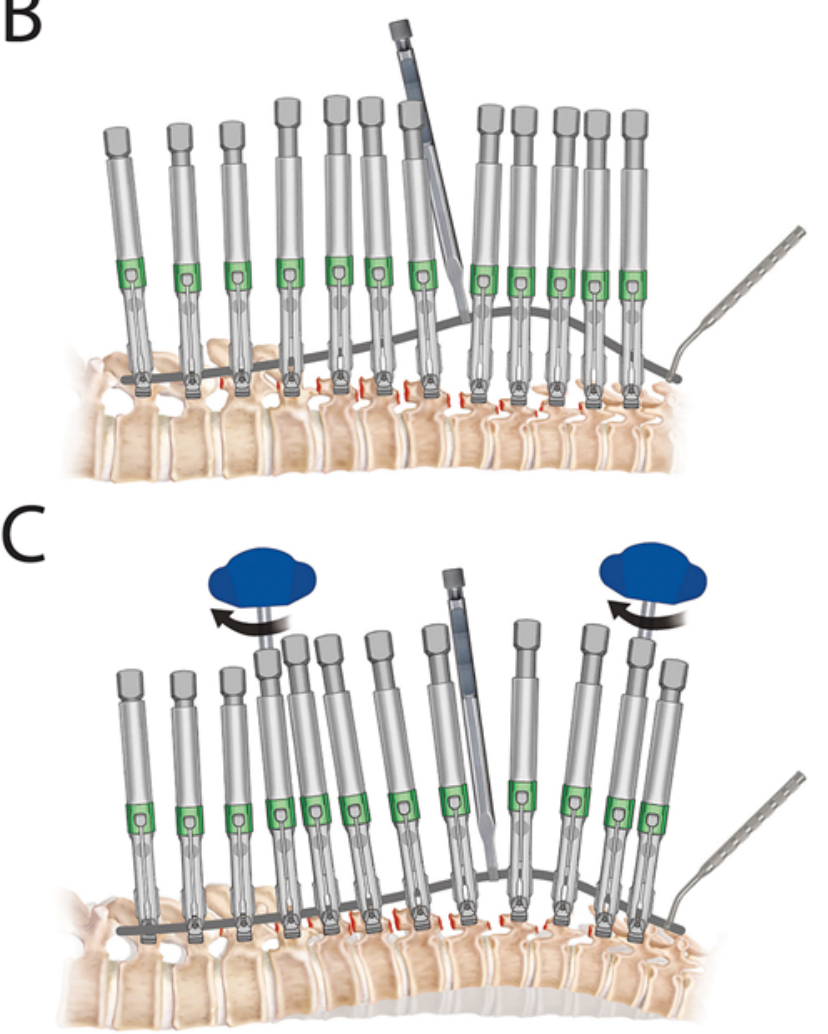

D

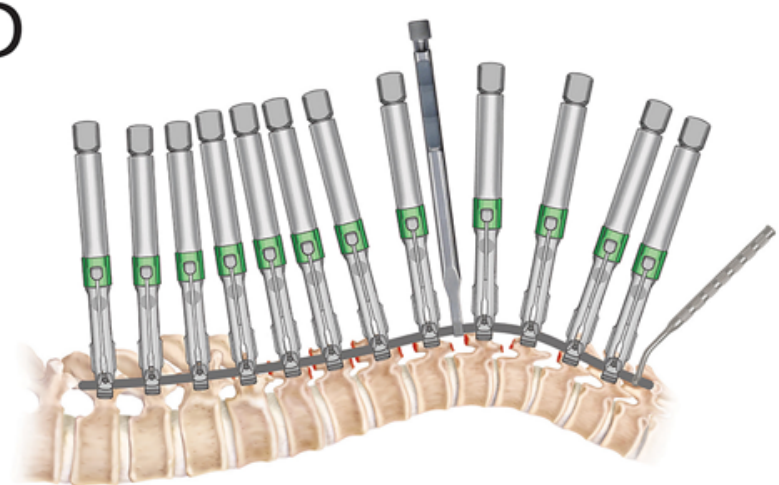

FIG. 2. Gradual sagittal translation and apex translocation via dual rods. Graphic representation of simultaneous translation with the dual contoured rod (ST2R) technique presented from posterior (A) and sagittal (B and C) views. A: Impression of placement reducers onto the pedicle screws, with inserted rods, along the spine. B: Both rods are held in place within the canal of the reducers by the assisting surgeon, with the contoured rods presenting an apex at the T4-6 level. C: From the most caudal and cranial site, reducers are gradually and bilaterally tightened toward the center, directing the rods into the pedicle screw, forcing the spine to take on the shape of the rods. D: After the reducers are fully screwed down to insert the rods into the pedicle screws along the entire instrument, the spine should have adopted an improved sagittal curvature presenting a TK apex approaching the T4-6 height. Copyright Daisuke Sakai. Published with permission.

ducers were applied, depending on the desired magnitude of translation. Next, two separate $6.0-\mathrm{mm}$ cobalt-chrome rods were contoured and cut to match the desired sagittal profile while ensuring that the length allowed for a maximum of $5 \mathrm{~mm}$ excess at each end of the most superior and inferior pedicle screws. Careful consideration was given to the curvature of the rod to establish the desired sagittal profile, with a specific modification to the original ST2R technique as described by Clement et al., ${ }^{10}$ but similar to that described by Faldini et al., ${ }^{4}$ by which we applied a sagittal contour that presented a convex aspect in the T4-6 range, which mirrors a healthy kyphosis profile. The contoured rods were inserted into the reducers in the cranialto-caudal direction throughout all segments for both concavity and convexity simultaneously (Fig. 1D and F).

While an assisting surgeon forcefully maintained the rods in place with vice grips, the primary surgeon gradually and bilaterally tightened the reduction handles on the reducers while continuously ensuring rod placement and orientation maintenance (Fig. 2A and B). Reduction was 


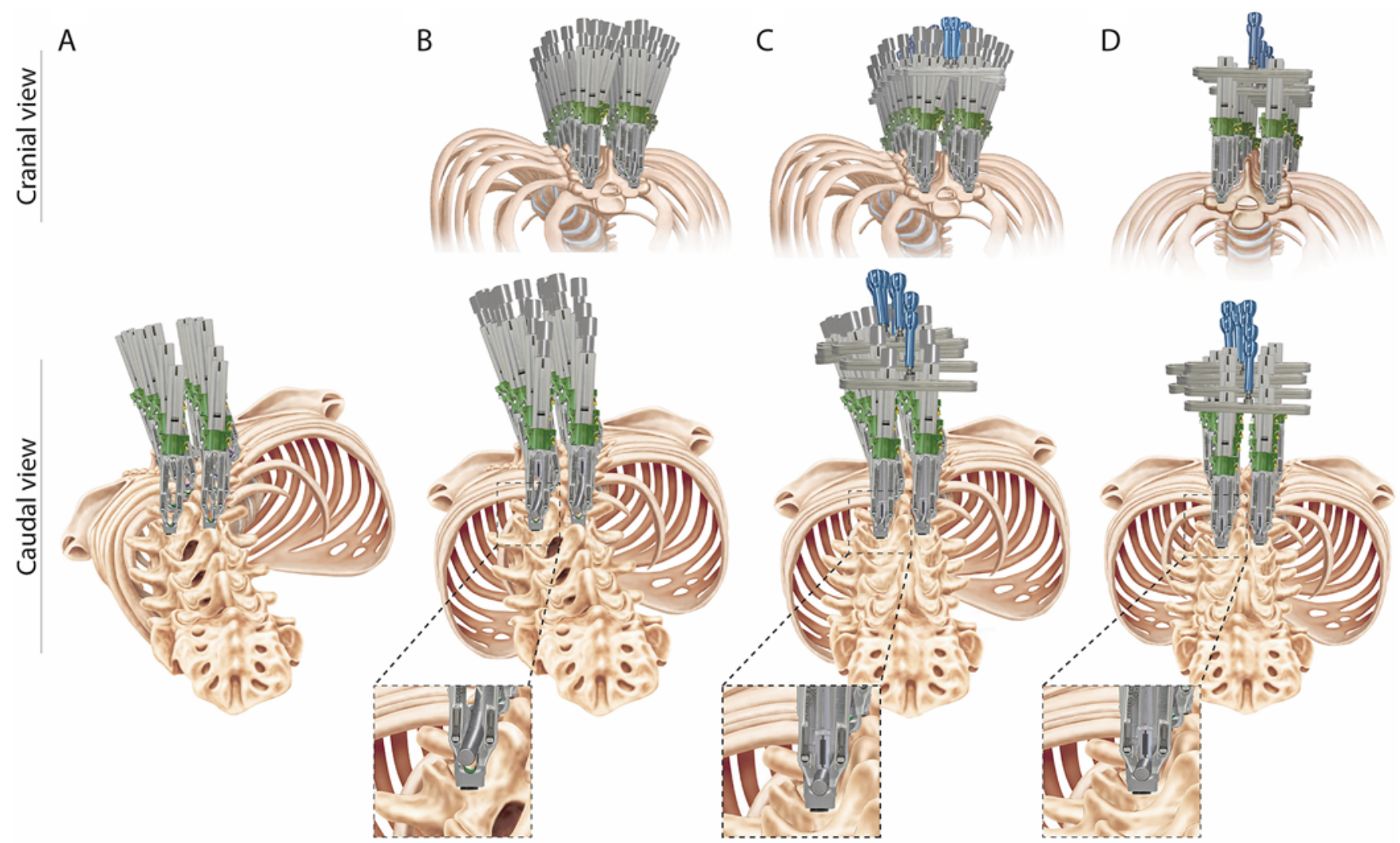

FIG. 3. Final axial adjustments with vertebral derotation device. Graphic representation of simultaneous translation with the dual contoured rod (ST2R) technique presented from an axial plane, from cranial (upper) and caudal (lower) views. A: Following the technique as illustrated in Fig. 1, the reducers are placed onto pedicle screws along the spine. B: Similar to the procedure description in Fig. 2, rods are bilaterally and simultaneously inserted along the reducer canal and gradually screwed down to lock the rods into the tulip-head screws, forcing the spine to take on the rod curvature profile. C: Finally, corrections are performed by linking each reducer pair with a connector. D: Using the connectors, after regionally unlocking the reducers, manual and local axial correction is applied along the spine. Also, distraction, particularly around the newly located apex levels, will also promote increase of TK. The engendered vertebral corrections are then locked in by securing the rods into place. Copyright Daisuke Sakai. Published with permission.

provoked by simultaneous tightening from the bottom and top of the device, toward the prospective apex, gradually altering the curvature of the spine, including spinal translation in the sagittal plane (Fig. 2C). Once the rods were fully settled in the screwheads, closure tops were inserted and screwed down to lock the rods into place (Figs. 2D and $3 \mathrm{~B}$ ). At a single vertebral level, compression and distraction adjustments were made by slightly untightening the to-be-readjusted levels and applying either a compressor or distractor to translocate the pedicle screws to closer or further proximity from each other, respectively.

Next, connectors for rotational correction were applied by connecting each of the reducer pairs (Fig. 3C). The final vertebral rotation corrections were applied on a regional basis, by slightly unfastening regional closure tops. While the secured reducers were held in place, another surgeon forcefully provoked rotation or additional translation of the spine toward the desired orientation to promote further corrections in the axial and coronal planes (Fig. $3 \mathrm{C}$ and $\mathrm{D})$. The correction was then locked into place by refastening the screw tops. This process was repeated for each vertebrae cluster until the vertebral column correction approached alignment with the midline. The correc- tions were confirmed by axial and coronal imaging. Finally, the connectors and rockets were detached, the screw tulips were clipped off if required, and the screw tops were checked and firmly secured. The surgical field was thoroughly irrigated, bone grafts were grafted to support spinal fusion, and the wound was sutured closed following standard procedures.

For the RR correction maneuver, the methods described in previously published work ${ }^{6,15-17}$ were followed. In short, surgical incision and articular process excision were performed as previously described. Next, the pedicle screws were inserted along the spine. The first $5.5-\mathrm{mm}$ titanium alloy-contoured rod was placed at the upper and lower anchor points and was gradually but forcefully rotated toward $90^{\circ}$. A second rod was inserted on the convex side and locked into place to secure the established spinal correction.

\section{Outcome Parameters}

Outcome parameters were assessed before and immediately after surgery, and 2-4 weeks, 1 year, and 2 years (final follow-up) after the operation. MRI was used preop- 


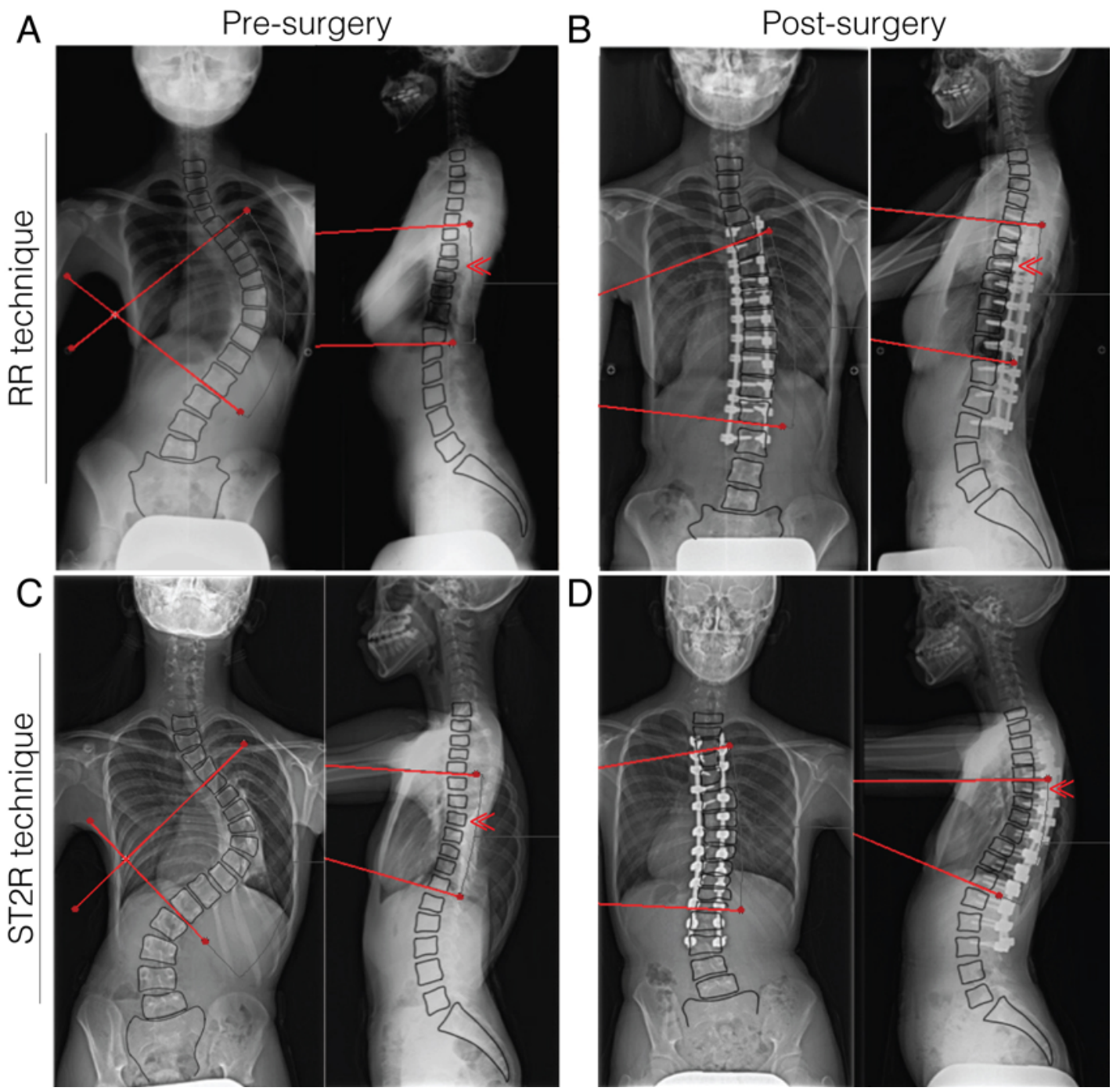

FIG. 4. A: A female AIS patient presented with abnormal curvature of the spine. The Cobb angle before the operation was $77.6^{\circ}$, the TK angle was $2.3^{\circ}$, and the apex was at level T8. B: Radiographic imaging at 2 years after the RR surgery showed marked improvement in the thoracic $\mathrm{Cobb}$ angle in the coronal plane $\left(27.1^{\circ}\right)$, although sagittal imaging revealed limited improvement as shown by a TK angle of $3.6^{\circ}$ and the apex at T8. C: A female AIS patient presented with a thoracic Cobb angle of $91.8^{\circ}$, TK angle of $12.6^{\circ}$, and the apex at level T8. D: Two years after the operation involving the ST2R technique, clinically significant improvement was observed in the coronal plane thoracic Cobb angle $\left(12.2^{\circ}\right)$. Additionally, improvement was observed in sagittal imaging, as shown by a TK angle of $23.9^{\circ}$ and translocation of the apex to T6. Images were processed by Adobe Illustrator. Red lines indicate the Cobb angle or TK measurements on the frontal or sagittal plane images. Red arrows indicate the determined TK apex level. Figure is available in color online only.

eratively to exclude any anomalies. Standard lateral and anteroposterior radiographic images were taken in the standing position for assessment of the main coronal Cobb angles, T5-12 TK angles, and LL for the L1-S1 region (Fig. 4A-D) The TK apex level was determined from sagittal images as the vertebral level apex of the TK. The correction rate was calculated as follows: [(preoperative Cobb angle - postoperative angle)/(preoperative Cobb angle)] $\times$ 100. The Cincinnati correction index (CCI) was applied following the original work of Vora et al. and was calculated by dividing the postoperative correction by the preoperative flexibility. ${ }^{18}$ Apical vertebral rotation (AVR) was determined according to Aaro and Dahlborn. ${ }^{19}$ Finally, improvement in clinical outcomes was examined through a Japanese version of the Scoliosis Research Society 22item questionnaire (SRS-22r). ${ }^{20}$

\section{Statistical Analysis and Data Management}

All data were collected, analyzed, and processed in Microsoft Excel. Statistical analysis was performed using GraphPad Prism. All numeric parameters were analyzed using two-way ANOVA with the Sidak multiple-comparison test after confirmation of a normal distribution unless stated otherwise. Changes in TK apex level were assessed using an unpaired t-test. All values are presented as mean \pm standard deviation. Differences were considered significant at $\mathrm{p}<0.05$. 


\section{Results}

The average patient ages were $14.1 \pm 2.4$ and $14.8 \pm 2.1$ years for the RR and ST2R cohorts, respectively. None of the preoperative values differed significantly between the RR and ST2R groups (Table 1). All surgical procedures (Figs. 1-3) were performed successfully without any major perioperative complications, i.e., no instrumentation failure or other surgical complications recorded. Intraoperative monitoring revealed no episodes of change to indicate major neurological deficits or other complications. Neither cohort reported any nonfusion cases. A minor wound infection was recorded in the ST2R cohort, which resolved after an antibiotic regimen, and one wound infection was recorded in the RR group, which was successfully resolved by wound debridement and antibiotics.

The coronal thoracic Cobb angle decreased significantly after the operation in both the RR and ST2R groups, from $55.3^{\circ} \pm 8.7^{\circ}$ to $21.8^{\circ} \pm 5.4^{\circ}$ for the RR group and from $56.8^{\circ} \pm 9.8^{\circ}$ to $15.8^{\circ} \pm 5.8^{\circ}$ in the ST2R group (Fig. 5 , Tables 1 and 2). The Cobb angle was significantly lower at each follow-up time point in the ST2R group than in the RR group (Fig. 5A). Similarly, the correction rate was significantly higher $(\mathrm{p}=0.004)$ for the ST2R group than for the RR group: $72.3 \% \pm 9.1 \%$ and $60.4 \% \pm 11.3 \%$, respectively (Fig. 5A and F). AVR decreased from pre- to postsurgery in both groups, from $16.4^{\circ} \pm 6.7^{\circ}$ to $15.1^{\circ} \pm$ $6.5^{\circ}$ for ST2R and from $17.0^{\circ} \pm 7.5^{\circ}$ to $15.6^{\circ} \pm 6.5^{\circ}$ for $\mathrm{RR}$, but this decrease did not significantly differ between the two cohorts (Fig. 5D, Tables 1 and 2).

The TK angle improved significantly throughout the study for both the RR and ST2R groups (Fig. 5B). Postoperatively, the TK angle was larger in the ST2R group $\left(27.0^{\circ}\right.$ $\left.\pm 2.7^{\circ}\right)$ than in the RR group $\left(5.9^{\circ} \pm 5.4^{\circ}\right)(\mathrm{p} \leq 0.001)$. A higher percentage of patients in the ST2R group were no longer hypokyphotic $\left(\mathrm{TK} \geq 20^{\circ}\right): 92 \%$ in the ST2R group compared with only $29.6 \%$ in the RR cohort.

The TK at the 1- and 2-year follow-ups was slightly decreased in the ST2R cohort, although these differences were no longer significant at the 2-year follow-up $(p=$ 0.056). However, there remained a clear trend toward clinically significant improvement in TK for the ST2R group (Fig. 5B, Table 2). The LL values did not show a clear improvement for either group, and the final angles were $44.6^{\circ}$ $\pm 13.0^{\circ}$ and $51.1^{\circ} \pm 8.8^{\circ}$ for the ST2R and RR groups, respectively (Fig. 5C). The CCI showed enhanced improvement in the ST2R group, although the difference was not significant compared with the RR group (Fig. 5G). The RH decreased significantly in both groups, although the established correction was maintained better after the operation in the ST2R group; the RH values were $6.5 \pm 2.7$ and $9.0 \pm$ $4.5 \mathrm{~cm}$ for the ST2R and RR groups, respectively (Fig. 5E).

The distribution of the TK apex ranged from T10 to $\mathrm{T} 7$ before the operation and did not differ significantly between the groups (Table 1). Following the surgical intervention, the apex moved slightly upward in the RR group (Fig. 6A) but did not improve in 2 of the 27 cases. By contrast, the TK apex improved significantly by $2.2 \pm$ 0.6 levels in the ST2R group (Fig. 6B) and approached a healthy apex range of T4-6 (Fig. 6A). The average change in apex translocation was significantly different between the groups $(\mathrm{p}<0.001)$ (Fig. 6B).
Clinical outcomes, as analyzed by SRS-22r, revealed a trend of improvement in sum scores for RR $(3.5 \pm 1.0$ to $3.8 \pm 0.7, \mathrm{p}=0.525)$ as well as for ST2R $(3.7 \pm 0.8$ to $4.1 \pm$ $0.6, p=0.297$ ) (Fig. 5H). The trend favored ST2R, but the difference was not significant $(\mathrm{p}=0.516)$. SRS-22r subcategory analysis (Table 2, Supplementary Fig. 1) revealed a significant improvement in patients treated with ST2R in function ( $p=0.0262)$, pain $(p=0.0134)$, and self-image ( $p$ $<0.001$ ) scores, while RR only engendered a significant improvement in self-image scores $(\mathrm{p}=0.001)$. Comparison of postoperative scores between cohorts revealed significantly enhanced improvements in pain $(\mathrm{p}=0.0364)$ and satisfaction $(p=0.008)$ categories, favoring the ST2R cohort.

\section{Discussion}

The aim of this investigation was to describe a new adaptation of the ST2R technique as reported by Clement et al. ${ }^{10}$ for thoracic hypokyphotic AIS patients. We focused on whether the ST2R technique would improve the TK apex location and outcomes relative to a case-matched cohort treated with RR.

However, one limitation of our study is the discrepancy of rods used in the RR and ST2R maneuvers, i.e., the $5.5-\mathrm{mm}$ titanium versus the $6.0-\mathrm{mm}$ cobalt-chrome alloy rods, respectively. This discrepancy is primarily due to the retrospective nature of the study and the consequential change in materials used over time in our practice. As such, outcome differences between the two techniques may be in part explained by the differences in the rods applied. Nevertheless, the roles of the rods in the RR and ST2R techniques are inherently different. RR uses a single rod to support a rotational correction of the spine at the concavity side, while a second rod is placed after the correction to provide support to preserve the correction. The ST2R technique involves two rods simultaneously to aid a translational correction of the spine, which distributes the forces over more rod material. Although it is generally assumed that stiffer rods might improve the spinal correction capacity, current data do not provide clear evidence in favor of this hypothesis. Numerous studies have examined the role of rod stiffness, for example, by increasing the rod diameter or changing the rod material. Generally, these studies failed to find clear or clinically relevant improvements in corrections with increased rod strength. In a large multicenter study by Prince et al., multifactor regression analysis showed that rod stiffness did not improve the spinal correction outcomes. ${ }^{21}$ Similar, but smaller, retrospective clinical studies have also been unable to demonstrate a clear benefit for kyphosis correction by enhanced rod stiffness. ${ }^{22-24}$ However, some small studies have reported indirect evidence of an association between rod stiffness and improved outcomes. ${ }^{25,26}$ Studies that have directly compared cobalt-chrome alloy rods to titanium alloy rods did not show a clear advantage of either rod material. ${ }^{22,24,27-29}$ Furthermore, cobalt-chrome alloys are more susceptible to losing their overall stiffness due to contouring and less able to maintain their shape, whereas titanium alloys tend to be more resilient for maintaining their profile upon implantation..$^{24,30,31}$ In our study, the ST2R meth- 
A

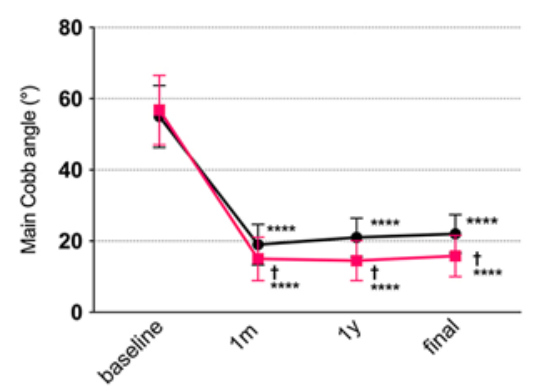

C

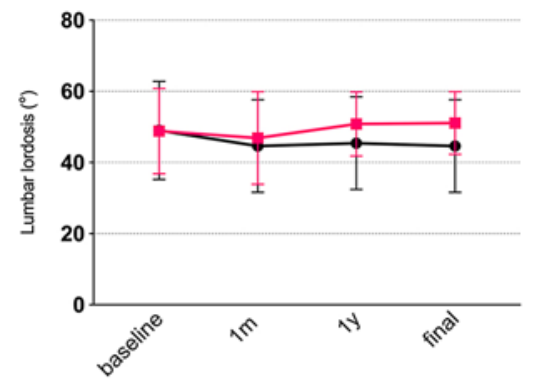

E

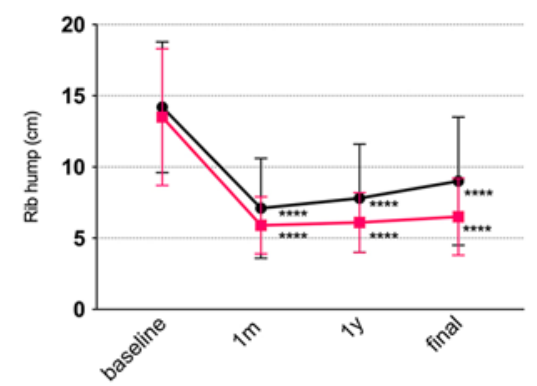

G

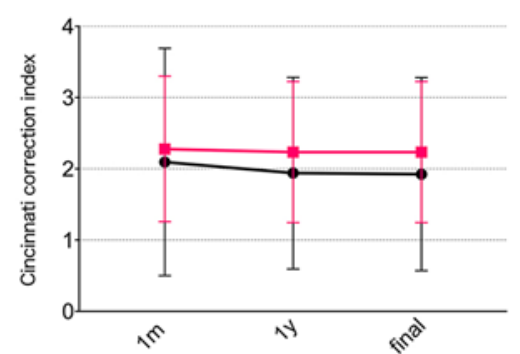

B

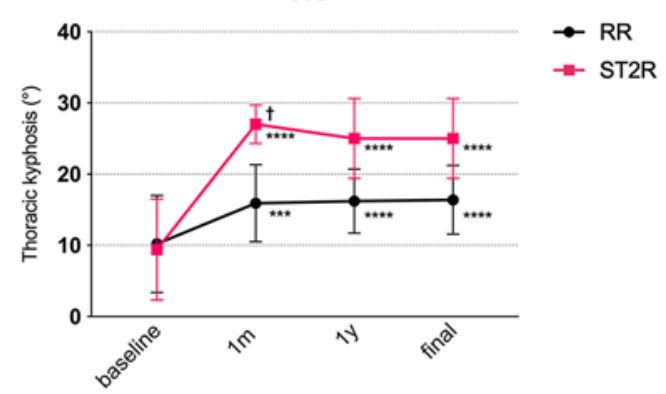

D

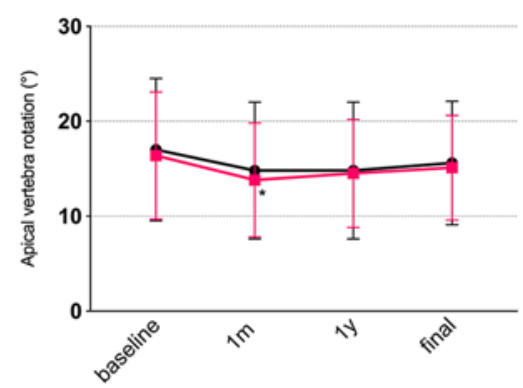

F

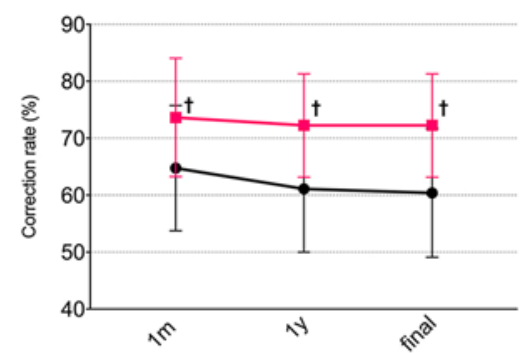

H

SRS-22r (sum)

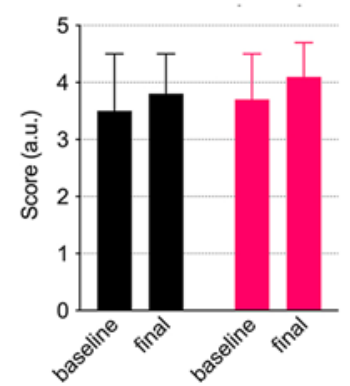

FIG. 5. Graphical representation of the clinical outcome parameters for patients treated with $R R$ (black, $n=27$ ) or ST2R (red, $n=$ $25)$ for adolescent idiopathic scoliosis patients. All values are presented as mean \pm SD. ${ }^{*}$, ${ }^{* *}$, and ${ }^{* * * *}$ indicate significant differences at $p<0.05, p<0.005$, and $p<0.001$, respectively, for the comparison with the baseline value for that treatment; $\dagger$ indicates a significant difference at $p<0.05$ for the ST2R compared with the RR group. Graphs demonstrate the main thoracic Cobb angle $(\mathbf{A})$, the TK angle (B), the LL angle (C), the AVR angle (D), the RH size (E), the correction rate $(\mathbf{F})$, the $\mathrm{CCl}(\mathbf{G})$, and the clinical outcomes as assessed by the SRS-22r (higher scores indicate alleviation of symptoms; for subcategory scores, see Supplementary Fig. 1) (H). Graphs were created with GraphPad Prism. a.u. = arbitrary units; $m=$ month; $y=$ year. Figure is available in color online only.

od involved the stiffer cobalt-chrome alloy; however, these rods were to a greater extent contoured, which thus likely reduced their rigidity. Whether and how these factors influenced our study remains unclear. However, considering that both cohorts consisted of young patients with flexible spines and relatively limited spinal deformities (predominantly Lenke type I), we believe that the differences in the rods likely had a minimal effect on our outcomes. An additional limitation of our study is that it involves a retrospective analysis of ST2R compared with an RR historical 
TABLE 2. Outcome parameters at the final follow-up

\begin{tabular}{|c|c|c|c|}
\hline & $\mathrm{RR}$ & ST2R & $\mathrm{p}$ Value \\
\hline Main thoracic Cobb angle, ${ }^{\circ}$ & $21.8 \pm 5.4$ & $15.8 \pm 5.8$ & 0.003 \\
\hline $\mathrm{TK}^{\circ}$ & $16.4 \pm 4.8$ & $25.2 \pm 5.6$ & 0.056 \\
\hline $\mathrm{LL},{ }^{\circ}$ & $44.6 \pm 13.0$ & $51.1 \pm 8.8$ & 0.146 \\
\hline $\mathrm{AVR}^{\circ}{ }^{\circ}$ & $15.6 \pm 6.5$ & $15.1 \pm 5.5$ & 0.995 \\
\hline $\mathrm{RH}, \mathrm{cm}$ & $9.0 \pm 4.5$ & $6.5 \pm 2.7$ & 0.089 \\
\hline Correction rate, $\%$ & $60.4 \pm 11.3$ & $72.3 \pm 9.1$ & 0.004 \\
\hline $\mathrm{CCl}$ & $1.9 \pm 1.4$ & $2.2 \pm 1.0$ & 0.724 \\
\hline SRS-22r (sum) & $3.8 \pm 0.7$ & $4.1 \pm 0.6$ & 0.516 \\
\hline SRS-22r (function) & $4.2 \pm 0.4$ & $4.5 \pm 0.5$ & 0.141 \\
\hline SRS-22r (pain) & $4.0 \pm 0.9$ & $4.6 \pm 0.3$ & 0.036 \\
\hline SRS-22r (self-image) & $3.3 \pm 0.4$ & $3.3 \pm 0.5$ & $>0.999$ \\
\hline SRS-22r (mental health) & $3.8 \pm 0.7$ & $4.1 \pm 0.5$ & 0.508 \\
\hline SRS-22r (satisfaction) & $3.5 \pm 0.7$ & $4.1 \pm 0.3$ & 0.008 \\
\hline
\end{tabular}

Average outcome measures for adolescent patients with hypokyphotic AIS treated with the RR technique or our adapted ST2R technique at the final (2-year) follow-up. Values are presented as mean \pm SD. Boldface type indicates statistical significance.

matched case-control group. The differences in the time periods of recruitment might have also affected the results, and thus require some discretion in interpretation. A future prospective randomized trial will be required to fully examine the ST2R potential.

Nonetheless, radiographic examination in the coronal plane demonstrated a significant improvement in the main Cobb angle for both ST2R and RR techniques, with correction rates of $72.3 \%$ and $60.4 \%$, respectively. These observations are consistent with previously published ST2R studies, including $73.7 \%$ improvement reported by Faldini et al. ${ }^{4}$ and $70 \%$ improvement reported by Clement and colleagues. ${ }^{32,33}$ Observation of the axial plane showed only a slight trend toward a reduction in AVR for the RR and
ST2R groups and no significant improvement in RH. The presurgery AVR values were low compared with the $25.3^{\circ}$ reported by Faldini et al. ${ }^{4}$ Sagittal plane measurements revealed greater improvement in the postoperative correction in the ST2R group than in the RR group, and hypokyphosis was relieved in a larger percentage of the ST2R group. However, the significant differences seen at the initial follow-up did not remain.

TK angles of $9.4^{\circ}$ for the ST2R group and $10.2^{\circ}$ for the RR group improved to $25.2^{\circ}$ and $16.4^{\circ}$, respectively. Clement and colleagues consistently demonstrated alleviation of hypokyphosis in all $10,11,32$ or 26 of 27 patients $^{33}$ by the ST2R technique and its later adaptations. Notably, their reported TK angles for the ST2R technique reached final
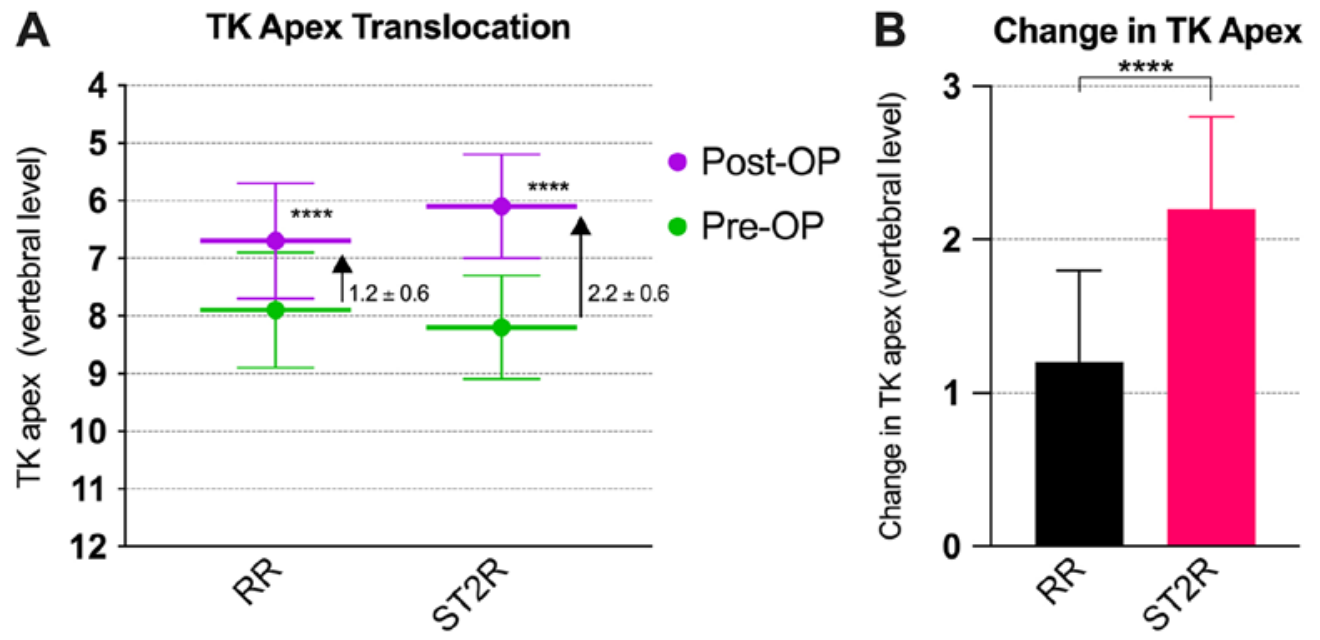

FIG. 6. Graphical representation of the TK apex determined in patients treated with the ST2R technique $(n=25)$ or with the RR maneuver $(n=27)$. Values represent the thoracic vertebra level. Graphs were created using GraphPad Prism. ${ }^{* * * *}$ indicates a significant difference at $p<0.001$. A: The TK apex vertebral level before and up to 2 months after surgery in the ST2R and RR groups; the level of significance refers to the comparison of the pre- and postoperative values. B: Change in the TK apex vertebral levels from before to after the operation; the level of significance refers to the change in the TK apex level in the ST2R group relative to that in the RR cohort. Figure is available in color online only. 
angles of $29^{\circ}-31.8^{\circ},{ }^{10,11,32,33}$ while we observed a maximum TK of $34.8^{\circ}$ at the final follow-up. Faldini et al. reported a TK range of $16.2^{\circ}-22.8^{\circ}$, although their findings were not derived within a hypokyphotic cohort. ${ }^{4}$

An additional observation is the ability to correct the TK apex more with the ST2R technique. Although Faldini et al. ${ }^{4}$ reported having improved the TK apex locations, they did not provide a quantification of this correction or a comparison to other techniques. Thus, our study is, to our knowledge, the first to present conclusive data on the ST2R technique's ability to translocate the TK apex by an average of 2.2 vertebral levels, thus resulting in a more natural sagittal profile (Fig. 4B and D), and thus significantly outperformed the RR technique (Fig. 6B).

The influence of the TK apex on physical function remains unknown, partly because of the limited reporting on the TK apex in AIS patients. Nevertheless, considering the influence of the apex on the spine shape, we believe the TK apex is likely a critical factor in determining the overall improvements in physical function and self-image. For example, work by Ito et al. in idiopathic scoliosis patients suggests a link between cervical kyphosis and a low TK apex $(<\mathrm{T} 10)$, which can alter the sagittal vertebral axis and promote forward-leaning spinal imbalance. ${ }^{34}$ Similarly, a TK apex < T10 was suggested to limit the compensatory function of the lumbar spine in a Scheuermann thoracolumbar kyphosis cohort. ${ }^{35}$ Work by Liu et al. in patients with ankylosing spondylitis treated by osteotomy found a relationship between the TK apex and enhanced correctional outcomes when the postsurgical TK apex was moved to T8 or above. ${ }^{12}$ Similarly, Lonner et al. found that postsurgery movement of the TK apex in adolescent patients with Scheuermann kyphosis improved SRS questionnaire outcomes. ${ }^{13}$ This notion is further supported by the enhanced improvements seen in the ST2R-associated SRS-22r outcomes, compared with the RR cohort. Taken together, these data suggest that the TK apex is an indicator for improvement in quality of life. As suggested by the outcomes of other studies of spinal balance compensation in the cervical and lumbar regions after thoracic intervention, ${ }^{9,34}$ it seems reasonable to expect that a mismatch in TK apex can provoke decompensation by the cervical or lumbar curve, e.g., cervical lordosis. Nevertheless, although a beneficial effect on clinical outcomes was confirmed, a direct effect of the TK apex position on, e.g., disability outcomes in AIS patients remains to be established.

Aside from the suggested advantages of the ST2R technique for spinal correction, it also has some practical advantages. Le Navéaux et al. ${ }^{36}$ recently demonstrated that significant flattening of the concave rod structure occurred directly after the vertebral manipulation and is a concern for both supporting and retaining the spinal correction. As ST2R involves dual rod application, the forces are distributed along more rod material and pedicle screws, thus reducing the stress at single points. ${ }^{11}$ This would likely reduce the risk of screw pullouts and rod flattening, thus preserving the translation ability of the correction maneuver. Finally, our described technique uses reducers also linked through connector segments to limit screw-rod friction and to improve ease of use. ${ }^{11,36}$ The original work by Clement et al. ${ }^{10}$ did not clearly identify the equipment used, which may have resulted in limited translation to the clinic. Later work by Faldini et al. used deformity reduction jacks (Crickets) to alleviate the screw-rod friction and to allow for dispersion of the applied forces as a vertebral rotation technique. ${ }^{4}$ Advances in instrumentation, such as Crickets and various reducers, have supported impressive triplanar corrections. Here, we report our application of commercially available reducers and present a comprehensive description of our surgical method with the aim of encouraging other spinal surgeons to adopt the ST2R technique, in particular for hypokyphotic AIS patients.

\section{Conclusions}

We have described our adaptation of the ST2R technique that allows for triplanar correction of the hypokyphotic spine in AIS patients. We found greater improvements in the main Cobb angle, TK, and clinical outcomes in the ST2R group than in the RR group. Our study is, to our knowledge, the first to report comprehensively on the ability to improve TK apex translocation. Given the commercial availability and common use of this surgical equipment, we encourage the adaptation of the ST2R technique for surgical correction of hypokyphosis in AIS patients.

\section{Acknowledgments}

We thank Medtronic plc (USA) for gifting the images used in Figs. 1-3 to illustrate the ST2R technique.

\section{References}

1. Konieczny MR, Senyurt H, Krauspe R. Epidemiology of adolescent idiopathic scoliosis. J Child Orthop. 2013;7(1):3-9.

2. Lafon Y, Lafage V, Dubousset J, Skalli W. Intraoperative three-dimensional correction during rod rotation technique. Spine (Phila Pa 1976). 2009;34(5):512-519.

3. Theruvath AS, Rajat MMG, Chhabra H, et al. Correction maneuvers in scoliosis surgery-an overview. Kerala J Orthop. 2012;25(2):73-77.

4. Faldini C, Perna F, Geraci G, et al. Triplanar correction of adolescent idiopathic scoliosis by asymmetrically shaped and simultaneously applied rods associated with direct vertebral rotation: clinical and radiological analysis of 36 patients. Eur Spine J. 2018;27(2)(suppl 2):165-174.

5. Emami A, Deviren V, Berven S, et al. Outcome and complications of long fusions to the sacrum in adult spine deformity: Luque-Galveston, combined iliac and sacral screws, and sacral fixation. Spine (Phila Pa 1976). 2002;27(7):776-786.

6. Newton PO, Yaszay B, Upasani VV, et al. Preservation of thoracic kyphosis is critical to maintain lumbar lordosis in the surgical treatment of adolescent idiopathic scoliosis. Spine (Phila Pa 1976). 2010;35(14):1365-1370.

7. Glassman SD, Bridwell K, Dimar JR, et al. The impact of positive sagittal balance in adult spinal deformity. Spine (Phila Pa 1976). 2005;30(18):2024-2029.

8. Hiyama A, Sakai D, Watanabe M, et al. Sagittal alignment of the cervical spine in adolescent idiopathic scoliosis: a comparative study of 42 adolescents with idiopathic scoliosis and 24 normal adolescents. Eur Spine J. 2016;25(10):3226-3233.

9. Smith TH. Adolescent idiopathic scoliosis: the mechanobiology of differential growth. JOR Spine. 2020;3(4):e1115.

10. Clement JL, Chau E, Kimkpe C, Vallade MJ. Restoration of thoracic kyphosis by posterior instrumentation in adolescent idiopathic scoliosis: comparative radiographic analysis of two 
methods of reduction. Spine (Phila Pa 1976). 2008;33(14): 1579-1587.

11. Clément JL, Chau E, Vallade MJ, Geoffray A. Simultaneous translation on two rods is an effective method for correction of hypokyphosis in AIS: radiographic results of 24 hypokyphotic thoracic scoliosis with 2 years minimum follow-up. Eur Spine J. 2011;20(7):1149-1156.

12. Liu ZJ, Qian BP, Qiu Y, et al. Does relocation of the apex after osteotomy affect surgical and clinical outcomes in patients with ankylosing spondylitis and thoracolumbar kyphosis? J Neurosurg Spine. 2019;31(1):27-34.

13. Lonner BS, Parent S, Shah SA, et al. Reciprocal changes in sagittal alignment with operative treatment of adolescent Scheuermann kyphosis - prospective evaluation of 96 patients. Spine Deform. 2018;6(2):177-184.

14. Lenke LG, Betz RR, Harms J, et al. Adolescent idiopathic scoliosis: a new classification to determine extent of spinal arthrodesis. J Bone Joint Surg Am. 2001;83(8):1169-1181.

15. Lowenstein JE, Matsumoto H, Vitale MG, et al. Coronal and sagittal plane correction in adolescent idiopathic scoliosis: a comparison between all pedicle screw versus hybrid thoracic hook lumbar screw constructs. Spine (Phila Pa 1976). 2007; 32(4):448-452.

16. Winter RB, Lovell WW, Moe JH. Excessive thoracic lordosis and loss of pulmonary function in patients with idiopathic scoliosis. J Bone Joint Surg Am. 1975;57(7):972-977.

17. Kim YJ, Lenke LG, Kim J, et al. Comparative analysis of pedicle screw versus hybrid instrumentation in posterior spinal fusion of adolescent idiopathic scoliosis. Spine (Phila Pa 1976). 2006;31(3):291-298.

18. Vora V, Crawford A, Babekhir N, et al. A pedicle screw construct gives an enhanced posterior correction of adolescent idiopathic scoliosis when compared with other constructs: myth or reality. Spine (Phila Pa 1976). 2007;32(17):1869_ 1874.

19. Aaro S, Dahlborn M. Estimation of vertebral rotation and the spinal and rib cage deformity in scoliosis by computer tomography. Spine (Phila Pa 1976). 1981;6(5):460-467.

20. Asher MA, Lai SM, Glattes RC, et al. Refinement of the SRS22 Health-Related Quality of Life Questionnaire Function domain. Spine (Phila Pa 1976). 2006;31(5):593-597.

21. Prince DE, Matsumoto H, Chan CM, et al. The effect of rod diameter on correction of adolescent idiopathic scoliosis at two years follow-up. J Pediatr Orthop. 2014;34(1):22-28.

22. Monazzam S, Newton PO, Bastrom TP, Yaszay B. Harms Study G. Multicenter comparison of the factors important in restoring thoracic kyphosis during posterior instrumentation for adolescent idiopathic scoliosis. Spine Deform. 2013;1(5): 359-364.

23. Huang TH, Ma HL, Wang ST, et al. Does the size of the rod affect the surgical results in adolescent idiopathic scoliosis? 5.5-mm versus 6.35-mm rod. Spine J. 2014;14(8):1545-1550.

24. Sia U, Tan BB, Teo YY, Wong CC. Post-implantation deformation of titanium rod and cobalt chrome rod in adolescent idiopathic scoliosis. Malays Orthop J. 2019;13(1):14-19.

25. Liu H, Li Z, Li S, et al. Main thoracic curve adolescent idiopathic scoliosis: association of higher rod stiffness and concave-side pedicle screw density with improvement in sagittal thoracic kyphosis restoration. J Neurosurg Spine. 2015;22(3): 259-266.

26. Abul-Kasim K, Karlsson MK, Ohlin A. Increased rod stiffness improves the degree of deformity correction by segmental pedicle screw fixation in adolescent idiopathic scoliosis. Scoliosis. 2011;6:13.

27. Angelliaume A, Ferrero E, Mazda K, et al. Titanium vs cobalt chromium: what is the best rod material to enhance adolescent idiopathic scoliosis correction with sublaminar bands? Eur Spine J. 2017;26(6):1732-1738.
28. Etemadifar MR, Andalib A, Rahimian A, Nodushan SMHT. Cobalt chromium-Titanium rods versus Titanium-Titanium rods for treatment of adolescent idiopathic scoliosis; which type of rod has better postoperative outcomes? Rev Assoc Med Bras (1992). 2018;64(12):1085-1090.

29. Sabah Y, Clément JL, Solla F, et al. Cobalt-chrome and titanium alloy rods provide similar coronal and sagittal correction in adolescent idiopathic scoliosis. Orthop Traumatol Surg Res. 2018;104(7):1073-1077.

30. Serhan H, Mhatre D, Newton P, et al. Would CoCr rods provide better correctional forces than stainless steel or titanium for rigid scoliosis curves? J Spinal Disord Tech. 2013;26(2): E70-E74.

31. Demura S, Murakami H, Hayashi H, et al. Influence of rod contouring on rod strength and stiffness in spine surgery. Orthopedics. 2015;38(6): 5 520-e523.

32. Clement JL, Chau E, Geoffray A, Suisse G. Restoration of thoracic kyphosis by simultaneous translation on two rods for adolescent idiopathic scoliosis. Eur Spine J. 2014;23(suppl 4): S438-S445.

33. Clement JL, Chau E, Geoffray A, Vallade MJ. Simultaneous translation on two rods to treat adolescent idiopathic scoliosis: radiographic results in coronal, sagittal, and transverse plane of a series of 62 patients with a minimum follow-up of two years. Spine (Phila Pa 1976). 2012;37(3):184-192.

34. Ito K, Imagama $\mathrm{S}$, Ito Z, et al. Analysis of cervical kyphosis and spinal balance in young idiopathic scoliosis patients classified by the apex of thoracic kyphosis. Eur Spine J. 2016; 25(10):3220-3225.

35. Zhu W, Sun X, Pan W, et al. Curve patterns deserve attention when determining the optimal distal fusion level in correction surgery for Scheuermann kyphosis. Spine J. 2019;19(9): $1529-1539$.

36. Le Navéaux F, Aubin C-E, Parent S, et al. 3D rod shape changes in adolescent idiopathic scoliosis instrumentation: how much does it impact correction? Eur Spine J. 2017;26(6): $1676-1683$.

\section{Disclosures}

The authors report no conflict of interest concerning the materials or methods used in this study or the findings specified in this paper.

\section{Author Contributions}

Conception and design: Sakai. Acquisition of data: Sakai, Hiyama, Katoh, Tanaka, Sato, Watanabe. Analysis and interpretation of data: Sakai, Schol. Drafting the article: Schol. Critically revising the article: all authors. Reviewed submitted version of manuscript: all authors. Approved the final version of the manuscript on behalf of all authors: Sakai. Statistical analysis: Schol. Administrative/technical/material support: Sakai, Hiyama, Katoh, Tanaka, Sato, Watanabe. Study supervision: Sakai, Sato, Watanabe.

\section{Supplemental Information}

\section{Online-Only Content}

Supplemental material is available with the online version of the article.

Supplementary Fig. 1. https://thejns.org/doi/suppl/10.3171/ 2020.8.SPINE201232.

\section{Correspondence}

Daisuke Sakai: Tokai University School of Medicine, Kanagawa, Japan.daisakai@is.icc.u-tokai.ac.jp. 